\title{
Shedding new light on visual snow syndrome
}

comorbidities
such as
migraine and
tinnitus were
prevalent
among
individuals
at the severe
end of the
[visual snow]
spectrum

Visual snow is a form of sensory disturbance in which an individual continuously perceives small dots throughout their visual field. This phenomenon was first described in 1995 but has only been characterized in detail in the past few years. Two recently published papers report on the largest studies of visual snow to date, which provide new insights into the clinical features and epidemiology of this condition.

In the first study, which was published in Neurology, Francesca Puledda and colleagues set out to better define the clinical spectrum of visual snow. "My co-authors, Christoph Schankin and Peter Goadsby, were the first to provide clinical criteria for visual snow in a seminal paper published in 2014," Puledda comments. "Since then, recognition of this condition has grown; however, it is still very common for affected patients to struggle in finding a diagnosis for their symptoms."

The study participants, who were recruited from a self-help group

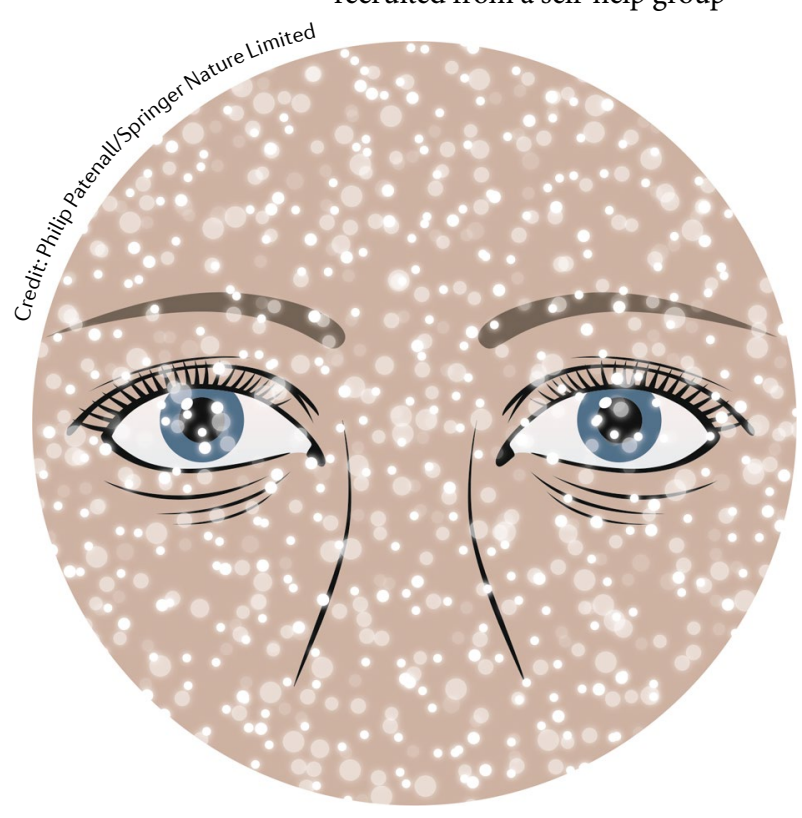

known as 'Eye on Vision', were asked to complete an online survey. The cohort included 1,104 patients with self-assessed visual snow, 1,061 of whom were identified as having visual snow syndrome (VSS). VSS is defined as visual snow plus at least two of the following symptoms: photophobia, entoptic phenomena (visual effects originating within the eye), palinopsia (prolongation of afterimages) and nyctalopia (poor vision in low light).

The researchers found that visual snow generally manifested in early life, and $\sim 40 \%$ of the participants had experienced the condition for as long as they could remember. Varying degrees of severity were reported, and comorbidities such as migraine and tinnitus were prevalent among individuals at the severe end of the spectrum.

"We think that describing the common presentation of visual snow will aid other researchers in recognizing the phenomenon, thus expanding interest in the disorder," concludes Puledda. "Our research efforts at the moment are centred on understanding more of the syndrome's basic biology, and we aim to look into the differences between visual snow and migraine in the near future."

The second study, led by Daniel Kondziella and published in the European Journal of Neurology, was prompted by an encounter with a patient with visual snow who seemed remarkably well informed about the condition. "It turned out that there is an entire community on the internet, consisting largely of people with self-diagnosed VSS," explains Kondziella. "Given the tremendous interest on social networks, I was wondering how frequent this syndrome was and how it had escaped medical attention until very recently."
To attempt to measure the true prevalence of visual snow, Kondziella and colleagues administered an online survey to 1,015 individuals from the general population in the UK. To avoid bias, the participants were simply told that the survey was about "the frequency of certain medical conditions", and they were not made aware of the primary purpose of the study. For this reason, the survey included control questions about diabetes and cleft lip.

Symptoms consistent with visual snow were reported by 38 participants (3.7\% of the cohort), and $22(2.2 \%)$ met the criteria for VSS. The individuals with visual snow had a mean age of 50.6 years, which was considerably higher than in the Puledda et al. study ( 29 years) and in previous studies of the phenomenon. The authors attribute this discrepancy to an increased tendency of younger individuals to seek medical attention and/or join internet self-help groups.

Kondziella argues that visual snow is a relatively benign condition and might even go unnoticed in many cases. "I think visual snow is a natural phenomenon, but most of us are simply not paying attention to it," he suggests. "Our visual impressions are edited, smoothed and adjusted, and visual noise is usually cancelled out; however, some people are so perceptive that they become aware of the noise."

Heather Wood

ORIGINAL ARTICLES Puledda, F. et al. Visual snow syndrome: a clinical and phenotypical description of 1,100 cases. Neurology https:// doi.org/10.1212/WNL.0000000000008909 (2020) | Kondziella, D. et al. Prevalence of visual snow syndrome in the UK. Eur. J. Neurol. https://doi.org/ 10.1111/ene.14150 (2020)

RELATED ARTICLES Liu, G. T. et al. Persistent positive visual phenomena in migraine. Neurology 45, 664-668 (1995)| Schankin, C. J. et al. “Visual snow": a disorder distinct from persistent migraine aura. Brain 137, 1419-1428 (2014) 